\title{
篹 \\ Reabilitação de tartaruga-cabeçuda (Caretta caretta Linnaeus, 1758) após traumatismo craniano
}

\author{
[Rehabilitation of loggerhead turtle (Caretta caretta Linnaeus, 1758) after head trauma]
}

\section{"Relato de Caso/Case Report"}

\author{
Amy Borges Moreira ${ }^{1,2,3}$, Fernanda Löffler Niemeyer Attademo ${ }^{1,2}$, Radan Elvis Matias de \\ Oliveira $^{1,2,4^{*}}$, Lucas Inácio dos Santos Melo ${ }^{2,4}$, Marcus Arthur Marques Dantas ${ }^{1,2}$, Augusto Carlos \\ da Bôaviagem Freire $^{1,2}$, Juliana Maia de Lorena Pires ${ }^{1,2}$, Heloisa Cristina de Morais e Sá Leitão ${ }^{1,2}$, \\ Iara Cecília da Costa Morais ${ }^{1,2}$, Flávio José de Lima Silva ${ }^{1,2,3,4,5}$
}

\begin{abstract}
${ }^{1}$ Centro de Estudos e Monitoramento Ambiental, Areia Branca-RN, Brasil.
${ }^{2}$ Projeto Cetáceos da Costa Branca, Universidade do Estado do Rio Grande do Norte (UERN), Mossoró-RN, Brasil.

${ }^{3}$ Programa de Pós-Graduação em Ciências Naturais, Faculdade de Ciências Exatas e Naturais, Universidade do Estado do Rio Grande do Norte (UERN), Mossoró-RN, Brasil.

${ }^{4}$ Laboratório de Morfofisiologia Animal Aplicada (LABMORFA), Programa de Pós-Graduação em Ciência Animal (PPGCA), Universidade Federal Rural do Semi-Árido (UFERSA), Mossoró-RN, Brasil.

${ }^{5}$ Programa de Pós-Graduação em Desenvolvimento e Meio Ambiente Associação Plena em Rede (PRODEMA), Universidade Federal do Rio Grande do Norte (UFRN), Natal-RN, Brasil.

*Autor para correspondência/Corresponding autor: E-mail: radan_elvis@ hotmail.com
\end{abstract}

\section{Resumo}

Traumas cranianos em tartarugas marinhas geralmente estão relacionados à ação antrópica por colisão com embarcações motorizadas. Em sua maioria, estes eventos são fatais, e quando não, várias sequelas podem permanecer. Desta forma, objetivou-se relatar um caso de uma tartaruga-cabeçuda (Caretta caretta) com politraumatismos craniano. O espécime foi resgatado encalhado no dia 17 de novembro de 2018, na praia de Retirinho, município de Aracati, Ceará, Brasil. O animal foi encontrado ativo e responsivo, com bom escore corpóreo e elevado grau de desidratação. Na inspeção, verificou-se uma lesão traumática na cabeça, que atingiu as placas frontoparietal, parietais e temporais esquerda e supraocular esquerda, com exposição de tecido mole adjacente. No ramo mandibular esquerdo, presença de lesão perfuro-incisa, e na cavidade oral, anzol de pesca aderido à musculatura da sínfise mandibular direita. No exame neurológico, observou-se excitação e hipersensibilidade ao toque, resposta ao teste de ameaça no olho direito, mas apresentou reflexo reduzido no olho esquerdo. Os quatro membros responderam satisfatoriamente ao teste de retirada, não manifestando movimentos incoordenados. O animal recebeu suporte terapêutico com administração de antibióticos, analgésicos, anti-inflamatórios, polivitamínicos, fluidoterapia e tratamento tópico. Após 291 dias de iniciado o processo de reabilitação, o animal foi reintroduzido em ambiente natural. Conclui-se que o protocolo terapêutico utilizado na reabilitação deste espécime de tartaruga-cabeçuda foi eficiente, principalmente no que diz respeito à terapia tópica. Além disso, a partir dos exames radiográficos foi possível visualizar a evolução do processo cicatricial das fraturas ósseas, auxiliando assim na tomada de decisão em liberar o animal à natureza.

Palavras-chave: Exame radiográfico; déficit neurológico; tratamento tópico; traumatismo.

\begin{abstract}
Head traumas in sea turtles are usually related to anthropic action by collision with motorized vessels. In the majority of the cases, these events are fatal, and when they are not, several sequelae may remain. Thus, this study aimed to report a case of treatment of a loggerhead sea turtle (Caretta caretta) with multiple head trauma. The specimen was rescued stranded in November $17^{\text {th }}$ of 2018 on Retirinho Beach, in the county of Aracati, Ceará State, Brazil. The animal was active, responsive, presented a good body score and a high degree of dehydration. Upon inspection, there was a head injury, which affected the frontoparietal, left parietals, left temporals and left supraocular scales, with exposure of soft tissue. In the left mandibular region, a perforated
\end{abstract}


lesion, and in the oral cavity, a hook adhered to the right mandibular symphysis, were also observed. During the neurological examination, hypersensitivity to touch was observed, the animal responded to the threat test on the right eye, but presented a reduced reflex in the left eye. All four limbs responded to the withdrawal test and the animal did not show uncoordinated movements. The animal received a therapeutic support with the administration of antibiotics, analgesics, anti-inflammatory drugs, polyvitamins, fluid therapy, and topical treatment. After 291 days of rehabilitation, the animal was reintroduced into a natural environment. In conclusion, the therapeutic protocol used in the rehabilitation of this loggerhead turtle specimen was efficient, especially with regard to the topical treatment. Furthermore, with the sequential follow-up through the radiographic examinations, it was possible to visualize the evolution of the healing process of the cranial bone fractures, thus assisting in the clinical decision for the release of the animal into its natural environment.

Keywords: radiographic examination; neurological deficit; topical treatment, trauma.

\section{Introdução}

Dentre as sete espécies de tartarugas marinhas existentes no mundo, cinco ocorrem no Brasil, incluindo o estado do Rio Grande do Norte, como área de importância para conservação de todas as cinco espécies (Farias et al., 2019; SilvaJúnior et al., 2019).

Uma das principais ameaças às tartarugas marinhas têm sido as atividades antrópicas (Farias et al., 2019; Rizzi et al., 2019), tais como ingestão de resíduos antropogênicos (Gall e Thompson, 2015), interação com a pesca (Gagliardi et al., 2018) e choque com embarcações motorizadas (Franchini et al, 2016). Por estes motivos, a tartaruga-cabeçuda (Caretta caretta) é classificada globalmente como "em perigo" de extinção (Butt et al., 2016).

Como consequências destas interações negativas, as tartarugas-marinhas têm apresentado politraumatismos e lesões cranianas (Goldberg et al., 2010). As consequências dos danos cerebrais secundários ao traumatismo cranioencefálico dependem do tipo de lesão, bem como da gravidade do trauma (Oertel et al., 2002). Este tipo de impacto gera grande preocupação, pois os traumatismos podem não levar apenas os animais à morte, mas deixar sequelas, como debilidade, desorientação, persistência de deficiência e, consequentemente, perda da capacidade reprodutiva, de forrageamento e de fuga (Goldberg et al., 2010).

Embora o diagnóstico precoce e o tratamento de indivíduos com traumatismo cranioencefálico melhorem a morbimortalidade associada a qualquer lesão intracraniana, para muitas tartarugas com esse diagnóstico em centros de reabilitação, o curso de ação mais humano é a eutanásia (Goldberg et al., 2010). A recuperação destes animais muitas vezes é difícil reverter, podendo deixar implicações permanentes, que os impedem de retornar para a natureza (Barten,
2006). Neste sentido, a padronização de técnicas, realização de manobras terapêuticas e a discussão de tratamentos veterinários fazem-se necessário, a fim de otimizar o tempo de recuperação e aumentar as chances de sobrevida destes animais. Desta forma, objetivou-se relatar um caso de reabilitação de uma tartaruga-cabeçuda com politraumatismos craniano.

\section{Descrição do Caso}

Uma tartaruga-cabeçuda, sub-adulta, sexo indefinido, foi resgatada encalhada no dia 17 de novembro de 2018 na praia de Retirinho (0440'28.3”'S; 037²4'15.6”W), no município de Aracati, estado do Ceará, Brasil. O registro e resgate do animal encalhado foi realizado pela equipe de Monitoramento Ambiental do Projeto Cetáceos da Costa Branca, da Universidade do estado do Rio Grande do Norte (PCCB-UERN). A execução destas atividades está autorizada pelo SISBIO-ICMBio (Licença n ${ }^{\circ}$ 13694-9).

$\mathrm{O}$ animal foi encontrado ativo e responsivo aos estímulos externos, cloacal e palpebral, com bom escore corpóreo $(39,6 \mathrm{~kg})$ e elevado grau de desidratação. Quanto aos dados morfométricos, o indivíduo apresentava $75,0 \mathrm{~cm}$ de comprimento curvilíneo da carapaça (CCC), 73,6 cm de largura curvilínea da carapaça (LCC).

No processo de estabilização foi realizado fluidoterapia com cloreto de sódio $(\mathrm{NaCl} 0,9 \%)$ (dose: $20 \mathrm{~mL} / \mathrm{Kg} / \mathrm{EV}$ - 30 gotas $/ \mathrm{min}$ ) para corrigir a desidratação do animal, além de analgésicos e anti-inflamatório (Tramal ${ }^{\circledR}$, dose: $5 \mathrm{mg} / \mathrm{Kg} / \mathrm{SC}$; Dexacorte ${ }^{\circledR}$, dose: $3 \mathrm{mg} / \mathrm{Kg} / \mathrm{EV}$ ) para o controle da dor. $\mathrm{O}$ animal foi mantido fora da água em um colchão, durante a reposição hidroeletrolítica, alimentação enteral e administração de medicamentos. Nesse momento, a contenção física era realizada segurando $o$ animal pelas extremidades da carapaça. Este manejo foi 
suficiente para manter o animal em condições satisfatórias para a realização dos procedimentos necessários.

No exame físico detalhado foram observadas algumas alterações, como na região da cabeça, uma lesão extensa que se estendia na placa frontoparietal, placas parietais esquerda, placa supraocular esquerda e placas temporais esquerda (Figura 1A), com extravasamento de líquido serosanguinolento e exposição de tecido mole adjacente. Na região mandibular esquerda havia outra lesão, perfuro-incisa (Figura 1B), e na cavidade oral, um anzol de pesca estava aderido à musculatura da sínfise mandibular direita, onde foi visualizado um abscesso caseoso e, posteriormente, uma fistula (Figura 1C). A remoção do anzol foi realizada sem sedação, apenas com contenção física do animal e bloqueio de anestésico local por infiltração na região de aderência.

O exame neurológico foi realizado com base na metodologia de Chrisman et al. (1997) adaptado para tartarugas marinhas. $\mathrm{O}$ exame foi realizado dentro e fora da água, sob posição de decúbito ventral e dorsal. Avaliou-se a atividade geral, postura de cabeça e corpo, movimento e coordenação de nadadeiras, força, tônus muscular e movimento da cauda. Também foram avaliados reflexos flexores das nadadeiras e nocicepção, resposta de endireitamento, reflexos dos nervos cranianos, reflexos cloacais e do pescoço, nocicepção da carapaça, cloaca e cauda. Dentre os sinais, observou-se hipersensibilidade ao toque, sugestivo de estímulo doloroso intenso. Apresentou uma boa resposta ao teste de ameaça no olho direito, mas reflexo reduzido no olho esquerdo. Todas as nadadeiras responderam ao teste de retirada, não sendo observados movimentos incoordenados. Quanto ao teste de deglutição, classificou-se como sem alterações. Já no teste olfativo essa avaliação é limitada em quelônios, não podendo obter resultados pertinentes.

$\mathrm{Na}$ primeira semana em reabilitação, a tartaruga foi mantida fora da água sobre um colchão, com o intuito de evitar maiores contaminações das lesões. Para evitar o ressecamento dos olhos, estes foram cobertos e umedecidos com solução fisiológica, não-estéril. Para evitar o ressecamento da carapaça e demais regiões foi utilizado óleo mineral, exceto em regiões de mucosas. Nesse primeiro período, a alimentação foi realizada via sonda esofágica, além da parenteral, utilizando-se um polivitamínico (Hertavita ${ }^{\circledR}$, dose: $1,4 \mathrm{~mL} / \mathrm{kg} / \mathrm{IV}$ a cada 24 horas). No tratamento terapêutico foram administrados $250 \mathrm{~mL}$ de fluidoterapia de $6 \%$ hidroxietilamido + cloreto de sódio (Voluven®) (dose: 5-10 $\mathrm{mL} / \mathrm{Kg} / \mathrm{EV}$ - 30 gotas/min) a cada 24 horas, durante 6 dias. $\mathrm{Na}$ terapia anti-inflamatória e analgésica foram utilizados: meloxicam (Maxicam ${ }^{\circledR}$, dose: $0,1 \mathrm{mg} / \mathrm{Kg} / \mathrm{EV} / \mathrm{SID} / 7$ dias), dexametasona (Dexacorte $\AA$ dose: 3 $\mathrm{mg} / \mathrm{Kg} / \mathrm{EV} / \mathrm{SID} / 3$ dias) e tramadol (Tramal ${ }^{\circledR}$, dose: $5 \mathrm{mg} / \mathrm{Kg} / \mathrm{SC} / \mathrm{a}$ cada 5 dias $/ 5$ aplicações). Para proteção gástrica, foi utilizado o omeprazol (Oprazon ${ }^{\circledR}, 4 \mathrm{mg} / \mathrm{Kg} / \mathrm{EV}$ ) durante 16 dias. A antibioticoterapia foi feita à base de ceftiofur (dose $2,2 \mathrm{mg} / \mathrm{Kg} / \mathrm{IM} / \mathrm{SID}$ ), durante 10 dias.

No tratamento tópico das feridas abertas, lesões do crânio, de mandíbula e fístula decorrente ao abscesso, foi realizada a limpeza da área afetada com solução fisiológica $(\mathrm{NaCl} 0,9 \%)$, e com antisséptico Lauril Dietileno Glicol Éter Sulfato de Sódio (Tergenvet ${ }^{\circledR)}$ ). Após a limpeza, foi aplicado cloridrato de oxitetraciclina + hidrocortisona spray (Terra-Cortril@)). Ao final, a lesão do crânio e da mandíbula foi coberta com gaze seca e aderida com esparadrapo. Após cinco dias de tratamento tópico da lesão craniana, não foi observada a presença de cáseos. Assim, no oitavo dia, foi realizado um curativo permanente com manta de vidro e resina epóxi revestindo todo o trauma do crânio (Figura 1D). Após procedimento, o animal foi colocado em um recinto com água salgada para observação (1 $\mathrm{m} 3=200 \mathrm{~cm}$ de diâmetro $\times 75 \mathrm{~cm}$ de profundidade), com temperatura da água entre $25^{\circ}$ $\mathrm{C}$ e $28^{\circ} \mathrm{C}$ e iluminação natural. Com o animal na água, iniciou-se as tentativas de alimentação espontânea com peixes e siris. A água salgada do recinto era trocada e abastecida diariamente, por meio de um sistema de motor bomba acoplado. Após a troca de água, o recinto era desinfetado com clorexidine $2 \%$ degermante ou hipoclorito de sódio $2,5 \%$.

Após 20 dias de reabilitação, o animal já apresentava uma melhora de sua condição física, mas ainda não apresentava interesse pelos alimentos ofertados. Entretanto, durante todo o período em que o animal não se alimentava, era realizada a alimentação por sonda esofágica a cada 24 horas, com $250 \mathrm{~g}$ de purê de peixe inteiros e 5 $\mathrm{mL}$ de emulsão de óleo de fígado de bacalhau (SCOTT®). O animal era contido pela carapaça sobre uma plataforma de 45 graus para cima, com o intuito de evitar o refluxo do alimento. A 
cavidade oral era aberta por meio de abre boca, por onde nela era introduzida uma sonda em direção ao esôfago até sua porção caudal.

No primeiro exame hematológico realizado do animal, foi verificado anemia microcítica hipocrômica, leucocitose com valores altos para heterófilos, linfócitos, basófilos e monócitos, e hipoproteinemia (Stacy et al., 2018; Deem et al., 2009). Para o tratamento da anemia, foi acrescentado $5 \mathrm{~mL}$ de Glicopan®, $5 \mathrm{~mL}$ de Hemolitan ${ }^{\circledR}$ e 3 gramas de Organew ${ }^{\circledR}$ na alimentação enteral. Esta alimentação era
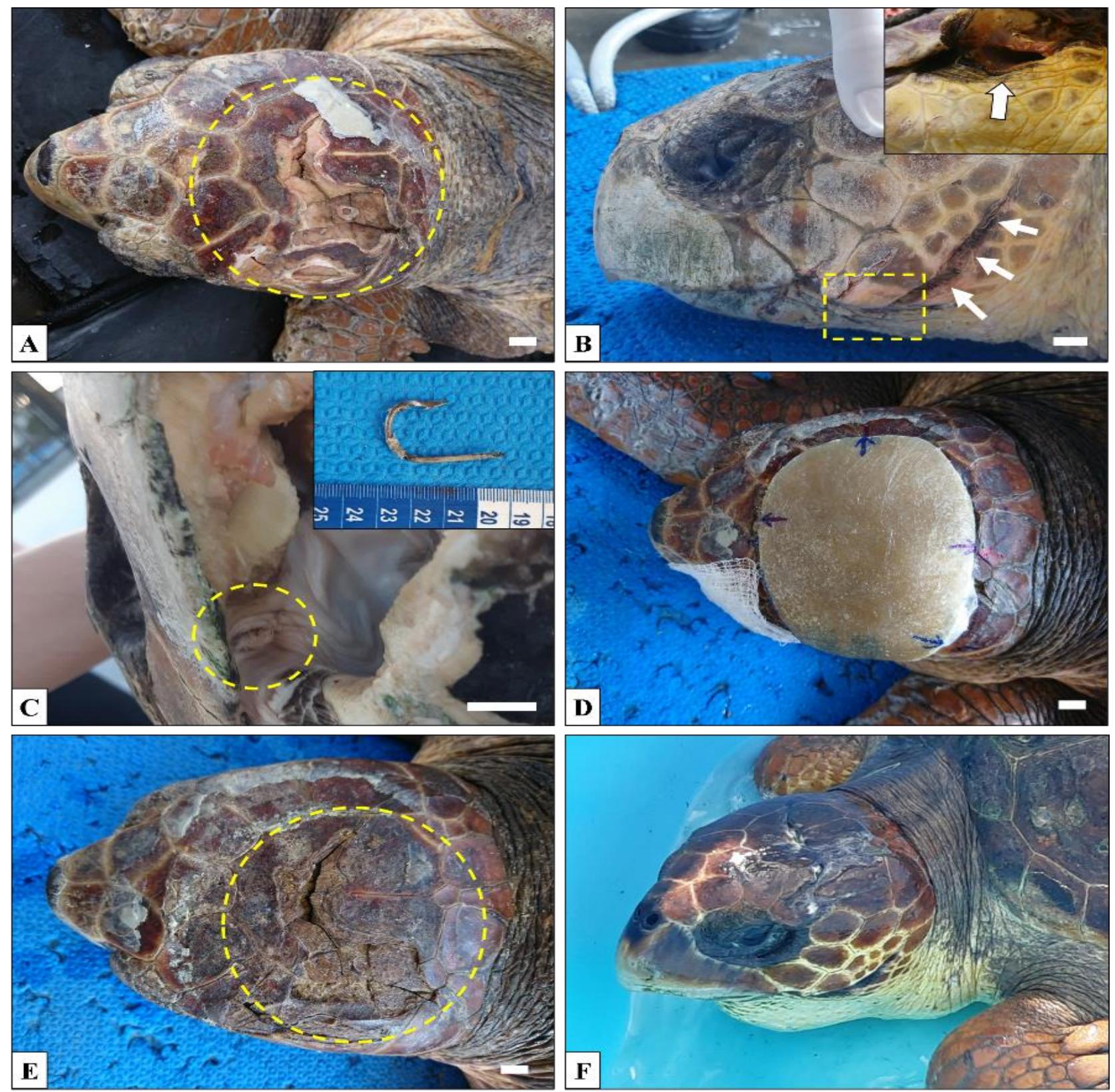

Figura 1. Lesões craniana em tartaruga-cabeçuda (Caretta caretta). (A) Lesão craniana com exposição de tecido mole (círculo tracejado). Barra: $1 \mathrm{~cm}$. (B) Lesão na região mandibular esquerda perfuro-incisa (setas). Barra: $1 \mathrm{~cm}$. (C) Abscesso fistulado na sínfise mandibular direita (círculo tracejado) decorrente de anzol de pesca (destaque na Figura C). Barra: $1 \mathrm{~cm}$. (D) Curativo permanente com manta de vidro e resina epóxi. Barra: $1 \mathrm{~cm}$. (E) Fratura em processo de cicatrização após 35 dias de tratamento (círculo tracejado). Barra: $1 \mathrm{~cm}$. (F) Fratura cicatrizada após 8 meses de tratamento. 
O primeiro exame radiográfico da região da cabeça foi realizado nas projeções dorsoventral e laterolateral esquerda. Foram observadas linhas de fratura com aparente desprendimento da parte calvaria, atingindo os ossos supra-occipital, parietal, frontal e pós-orbital esquerdo, com consequente descontinuidade em órbita esquerda (Figura 2A e 2B).

Completado três meses de reabilitação, com animal mantido em água, apresentando funções gastrointestinais normais e comportamento ativo, mas apresentava dificuldades para submergir e, por vezes, demonstrava lateralização do corpo e ruídos respiratórios durante repertório respiratório. Deste modo, foram administradas 10 aplicações de 4,5 $\mathrm{mL}$ de ceftazidima (30 mg/Kg/IM/ a cada $72 \mathrm{~h}$ ), devido os sinais de afecção respiratória. Nos dias subsequentes ao tratamento foi observada uma melhora clínica significativa, demonstrado em quadro de normalidade de natação, submersão e ausência de ruídos respiratórios.

O segundo exame hematológico foi realizado no dia 06 de maio de $2019\left(170^{\circ}\right.$ dia de reabilitação) após o animal apresentar um quadro de apatia, onde foi verificado anemia microcítica hipocrômica, além de leucocitose, valores altos para basófilos e hipoproteinemia. Em virtude aos achados, mais uma vez, a tartaruga foi submetida à antibioticoterapia. Neste caso, foi realizada uma associação de amicacina $(2,5 \mathrm{mg} / \mathrm{kg} / \mathrm{a}$ cada $48 \mathrm{~h} / \mathrm{EV} /)$ e ampicilina $(20 \mathrm{mg} / \mathrm{kg} / \mathrm{SID} / \mathrm{EV})$, durante a fluidoterapia com $(\mathrm{NaCl} 0,9 \%)$, no período de 7 dias, além de suplementação de tiamina (25 $\mathrm{mg} / \mathrm{kg} / \mathrm{SID}$ ) junto aos peixes, ofertado de forma assistida durante 15 dias. Nesse período, o animal não necessitava de alimentação entérica.

Um segundo exame radiográfico foi realizado no dia 14 de maio de $2019\left(178^{\circ}\right.$ dia de reabilitação) para o acompanhamento do processo de cicatrização do trauma, quando foi verificado linhas de fratura com aparente desprendimento de parte da calvária, atingindo ossos supra-occipital, parietal, frontal e pós orbital esquerdos, com consequente descontinuidade em órbita. Em comparação ao exame anterior (06/02/2019), menos linhas de fratura aparentes foram observadas e moderada reabsorção óssea em foco de fratura dorsal (Figura 2C e 2D).

A partir do dia 09 de junho de 2009 (204 ${ }^{\circ}$ dia de reabilitação), foi observada uma evolução clínica satisfatória do animal, com melhoria da atividade motora, natação e submersão no recinto. Também foi registrada uma melhora na resposta ocular do olho esquerdo. A partir do dia 16 de junho, o animal começou a apresentar maior interesse pela alimentação espontânea, e começou a apresentar comportamento de forrageamento no fundo do recinto. Após oito meses de reabilitação foi removido o curativo fixo de resina epóxi, observando completa cicatrização da fratura craniana descrita (Figura 1F).

No dia 28 de agosto de 2009 (284 ${ }^{\circ}$ dia de reabilitação), foi realizado o terceiro exame radiográfico da cabeça para confirmação do processo cicatrização (Figura 2E e 2F). No exame não foram verificados a descontinuidade em órbita e o desprendimento da calvária. As linhas de fratura estavam menos aparentes, e da mesma forma, sinais de reabsorção óssea não foram observados, confirmando o processo de consolidação.

Após 291 dias de reabilitação (9 meses e 16 dias), no dia 04/09/2019, o animal foi reavaliado pela equipe médica-veterinária e considerado apto para soltura em ambiente natural, sendo avaliado parâmetros fisiológicos (interesse alimentar, regime regular de excretas e bom escore corpóreo), clínicos (perfil hematológico dentro da normalidade para a espécie) e comportamentais (alimentação e forrageio submerso e exploração de todo o recinto de forma dinâmica) compatíveis para a espécie. Antes de proceder com a soltura, duas anilhas metálicas com identificação individual foram inseridas em ambas as nadadeiras anteriores (esquerda: BRA17517; direita: BRA17518). Após soltura, foi instituído um protocolo de alerta para a equipe de monitoramento de praia, no período de 30 dias, com extensão de $300 \mathrm{Km}$ de área monitorada. Durante esse período não foi identificado o encalhe do animal.

\section{Discussão}

Tartarugas marinhas acometidas por trauma relacionado à ação antrópica por colisão com embarcações, tem ocorrido em todo o mundo (Goldberg et al., 2010; Franchini et al., 2016). As tartarugas com trauma craniano chegam geralmente na costa sem vida ou bastante debilitadas, dificultando a reabilitação do animal (Goldberg et al., 2010). Farias et al. (2019) analisaram 4.760 tartarugas marinhas no Rio Grande do Norte, verificando que 55,27\% haviam encalhado com marcas de interação com a pesca e $4,15 \%$ destes com lesões por choque com embarcações. No presente estudo, além da lesão no crânio, o animal possuía um anzol na boca, 
sugerindo assim que a interação com a pesca possa ter ocorrido primariamente e, em seguida a lesão no crânio, a qual pode ter ocorrido por choque com embarcação ou golpe com objetos tipo remo ou outros.
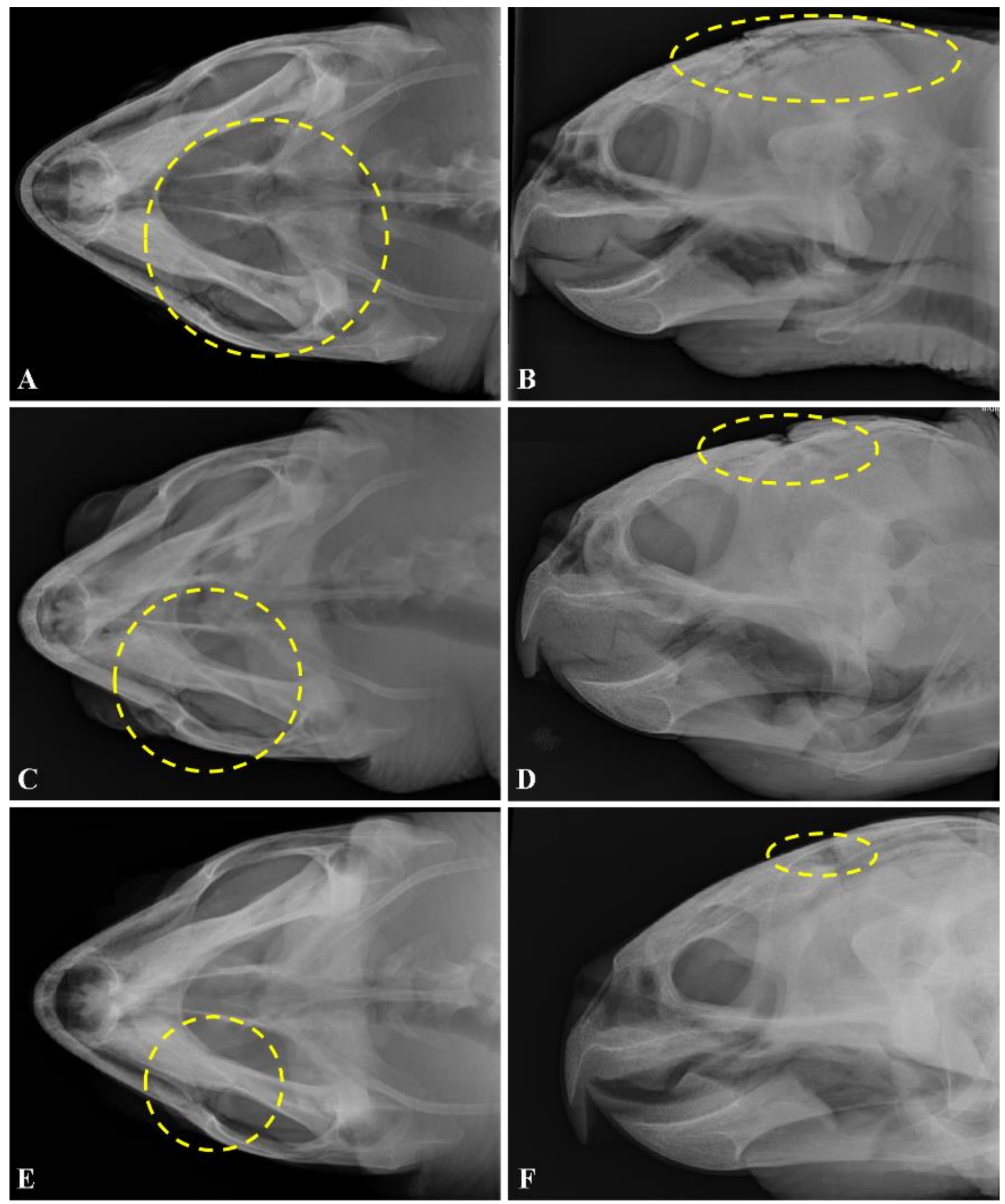

Figura 2. Exames sequenciais de radiografias de lesão craniana em tartaruga-cabeçuda (Caretta caretta) com projeções dorsoventral e laterolateral esquerda. (A e B) primeiro exame: Observou-se linhas de fratura com aparente desprendimento de parte da calvária, atingindo ossos supra-occipital, parietal, frontal e pós orbital esquerdos, com consequente descontinuidade em órbita (círculos tracejados). (C e D) segundo exame: verificou-se linhas de fratura menos evidentes quando comparadas ao exame anterior (06/02/2019) e moderada reabsorção óssea em foco de fratura dorsal (círculos tracejados). (E e F) terceiro exame: Neste último exame não foram verificados a descontinuidade em órbita e o desprendimento da calvária. As linhas de fratura estavam menos aparentes, e não foram observados sinais de reabsorção óssea, confirmando o processo de consolidação (círculos tracejados). 
As consequências do dano cerebral secundário ao traumatismo craniano dependem do tipo de lesão e da gravidade do trauma (Oertel et al., 2002). O trauma por impacto geralmente resulta em lacerações graves, fraturas do crânio e da carapaça, ou até mesmo amputação de membros (Orós et al., 2005). O traumatismo craniano grave pode causar lesões cerebrais e danificar as glândulas de sal, afetando assim a homeostase do animal (George, 1997). Apesar do traumatismo craniano deste relato de caso ter sido muito grave, não foi observado comprometimento significativo do sistema nervoso motor no exame neurológico. Contudo, há possibilidade da lesão ter comprometido alguns pares de nervos cranianos, com consequente mudança de comportamento ou déficit visual, olfativo ou do reflexo de deglutição que justifiquem a perda de interesse na alimentação e recuperação lenta do animal.

Protocolos de tratamento para feridas devem prevenir infecções, remover o exsudato e promover a cicatrização (Franchini et al., 2016). Contudo, a cicatrização de répteis difere da dos mamíferos, pois a produção de tecido de granulação é rara e a migração epitelial ocorre sob uma crosta proteica ou tecido epidérmico e dérmico necrótico (Keller et al., 2014). Por este motivo, é justificável o longo período no processo de cicatrização da lesão craniana da tartaruga deste caso.

Um estudo realizado por Smith e Barker (1988) avaliando os efeitos de vários curativos tópicos em feridas experimentais em cobras mostrou que o filme de poliuretano produzia os melhores resultados, além de evitar a contaminação de microrganismos, enquanto o pó e a pomada a base de antibióticos tópicos pareciam retardar a resposta cicatricial. Seguido por este raciocínio, na tartaruga desse relato, optou-se pelo curativo permanente com manta de vidro e resina epóxi, com o intuito de proteger a ferida e evitar contaminação, e assim, pode-se manter o animal dentro do recinto com água, durante todo o tratamento. Na literatura, há uma escassez de referências de tratamentos tópicos de lesões traumáticas em tartarugas marinhas, neste sentido, discussões de tratamentos tópicos se tornam importantes, a fim de comparar diferentes protocolos.

Lesões de grande complexidade e exposição de cavidade, conforme a descrita neste relato de caso, se não tratada, podem levar o animal a um processo infeccioso e óbito. Por outro lado, estudos com tartarugas marinhas, tanto de vida livre quanto de cativeiro, tem demonstrado que estes animais apresentam grande resistência a antibióticos (Foti et al., 2009; Al-Bahry et al., 2012; Blasi et al., 2020). Por este motivo, recomenda-se que antes de iniciar a antibioticoterapia, deve-se realizar a identificação do microrganismo por meio de cultura ou hemocultura, seguido do antibiograma. Em contrapartida, nesse estudo de caso, não realizamos as culturas/hemoculturas e antibiogramas, ao invés disso, optamos na utilização de antibióticos de amplo espectro de ação. Desta forma, a não realização da cultura e antibiograma pode ter implicado no tempo de recuperação do animal deste relato.

A radiografia digital, geralmente é a primeira escolha de exames de imagem, para diagnósticos em tartarugas marinhas, pois em geral, possui maior facilidade de acesso na maioria dos locais e resultados bastantes elucidários (Di Bello et al., 2013; Oliveira et al., 2020). O presente estudo corrobora com esta informação, uma vez que esta ferramenta permitiu o acompanhamento da evolução da lesão e auxiliou à equipe veterinária na tomada de decisão sobre o tratamento clínico a ser prescrito. Contudo, lesões intracranianas são melhores avaliadas por meio da tomografia computadorizada (TC), além de ser um método de imagem rápido e viável para tartarugas marinhas (Oliveira et al., 2012; Spadola et al., 2016; Oliveira et al., 2020). O uso da TC pode reduzir o tempo de tratamento, maximizar as chances de reabilitação bem-sucedida e, consequentemente, reduzir o custo associado à manutenção do animal em cativeiro (Valente et al., 2007; Erlacher-Reid et al., 2013; Spadola et al., 2016; Oliveira et al., 2020). No caso deste estudo, não foi possível realizar a TC no animal, devido à logística inviável do transporte do animal até o local mais próximo para realização do exame, totalizando em média $800 \mathrm{~km}$ a ser percorrido, o que poderia agravar o estado de saúde do animal.

\section{Conclusão}

O protocolo terapêutico utilizado na reabilitação deste espécime de tartaruga-cabeçuda foi eficiente, principalmente no que diz respeito à terapia tópica, e que, com o acompanhamento sequencial do trauma craniano por meio de exames radiográficos foi possível visualizar a evolução do processo cicatricial das fraturas ósseas, auxiliando assim na decisão clínica de liberação do animal na natureza. Independentemente da opção de tratamento escolhida, o animal não deve sentir dor 
ou desconforto e, se isso não for possível, deve-se considerar a eutanásia. Vale ressaltar que o protocolo terapêutico pode ser adaptado para atender casos individuais.

\section{Agradecimentos}

Agradecemos aos colaboradores do PCCBUERN que auxiliam informando a presença dos animais nas praias e aos tratadores e voluntários do PCCB-UERN, pela valiosa ajuda durante os tratamentos realizados.

\section{Conflito de Interesse}

Os autores declaram que não há conflitos de interesse.

\section{Referências}

Al-Bahry, S.N.; Al-Zadjali, M.A.; Mahmoud, I.Y.; Elshafie, A.E. Biomonitoring marine habitats in reference to antibiotic resistant bacteria and ampicillin resistance determinants from oviductal fluid of the nesting green sea turtle, Chelonia mydas. Chemosphere, 87(11): 13081315, 2012.

Barten, S.L. Shell damage. In: Mader, D.R. Reptile medicine and surgery. $2^{\text {nd }}$ ed. Florida: Marathon, 2006. p.893-899.

Blasi, M.F.; Migliore, L.; Mattei, D.; Rotini, A.; Thaller, M.C.; Alduina, R. Antibiotic resistance of gram-negative bacteria from wild captured loggerhead sea turtles. Antibiotics, 9(162): 1$11,2020$.

Butt, N.; Whiting, S.; Dethmers, K. Identifying future sea turtle conservation areas under climate change. Biological Conservation, 204B(1): 189-196, 2016.

Chrisman, C.L.; Walsh, M.; Meeks, J.C.; Zurawka, H.; LaRock, R.; Herbst, L.; Schumacher, J. Neurologic examination of sea turtles. Journal of the American Veterinary Medical Association, 211(8): 1043-1047, 1997.

Deem, S.L.; Norton, T.M.; Mitchell, M.; Segars, A.; Alleman, A.R.; Cray, C.; Poppenga, R.H.; Dodd, M.; Karesh, W.B. Comparison of blood values in foraging, nesting, and stranded loggerhead turtles (Caretta caretta) along the coast of Georgia, USA. Journal of Wildlife Diseases, 45(1): 41-56, 2009.

Di Bello, A.; Valastro, C.; Freggi, D.; Lai, O.; Crescenzo, G.; Franchini, D. Surgical treatment of injuries caused by fishing gear in the intracoelomic digestive tract of sea turtles. Diseases of Aquatic Organisms, 106(2): 93102, 2013.
Erlacher-Reid, C.D.; Norton, T.M.; Harms, C.A.; Thompson, R.; Reese, D.J.; Walsh, M.T.; Stamper, M.A. Intestinal and cloacal strictures in free-ranging and aquarium-maintained green sea turtles (Chelonia mydas). Journal of Zoo and Wildlife Medicine, 44(2):408-429, 2013.

Farias, D.S.; Alencar, A.E.B.; Bomfim, A.C.; Fragoso, A.B.L.; Rossi, S.; Moura, G.J.B.; Gavilan, S.A.; Silva, F.J.L. Marine turtles stranded in northeastern Brazil: composition, spatio-temporal distribution, and anthropogenic interactions. Chelonian Conservation and Biology, 18(1): 105-111, 2019.

Franchini, D.; Cavaliere, L.; Valastro, C.; Carnevali, F.; Van-Der-Esch, A.; Lai, O.; Di Bello, A. Management of severe head injury with brain exposure in three loggerhead sea turtles Caretta caretta. Diseases of Aquatic Organisms, 119(2): 145-152, 2016.

Foti, M.; Giacopello, F.M.; Bottari, C.; Fisichella, V.; Rinaldo, D.; Mammina, C. Antibiotic resistance of gram negatives isolates from loggerhead sea turtles (Caretta caretta) in the central Mediterranean Sea. Marine Pollution Bulletin, 58(9): 1363-1366, 2009.

Gagliardi, T.R.; Lopes, T.C.; Serafini, T.Z. Interação de tartarugas marinhas e a pesca no Brasil: Uma revisão da literatura. Arquivo de Ciências do Mar, 51(1): 101-124, 2018.

Gall, S.C.E.; Thompson, R.C. The impact of debris on marine life. Marine Pollution Bulletin, 92(1):170-179, 2015.

George, R.H. Health problems and diseases of sea turtles. In: Lutz, P.L.; Musick, J.A. The biology of sea turtles. Florida: CRC, 1997. p.363-386.

Goldberg, D.W.; Adeodato, A.; Almeida, D.T.; Corrêa, L.G.; Wanderlinde, J. Green turtle head trauma with intracerebral hemorrhage: Image diagnosis and treatment. Ciência Rural, 40(11): 2402-2405, 2010.

Keller, K.A.; Paul-Murphy, J.; Weber, S.; Kass, P.H.; Guzman, D.S.M.; Park, S.A.; Raghunathan, K.; Gustavsen, K.; Murphy, C.J. Assessment of platelet derived growth factor using a splinted full thickness dermal wound model in bearded dragons (Pogona vitticeps). Journal of Zoo and Wildlife Medicine, 45(4): 866-874, 2014.

Oertel, M.O.; Kelly, D.F.; McArthur, D.; Boscardin, W.J.; Glenn, T.C.; Lee, J.H.; Gravori, T.; Obukhov, D.; McBride, D.Q.; Martin, N.A. Progressive hemorrhage after head trauma: predictors and consequences of 
the evolving injury. Journal of Neurosurgery, 96(1): 109-116, 2002.

Oliveira, J.F.; Rossi Junior, J.L.; Leite, F.L.G.; Oliveira, D.C.; Costa, L.A.V.S.; Silva, I.C.C.; Teixeira, M.W.; Costa, F.S. Densitometria da vertebra dorsal, osso pleural e osso neural em tartarugas verdes higidas por tomografia computadorizada quantitative. Ciência Rural, 42(8):1440-1445, 2012.

Oliveira, R.E.M.; Attademo, F.L.N.; Moura, C.E.B.; Araujo Junior, H.N.; Costa, H.S.; Reboucas, C.E.V.; Silva, F.J.L.; Oliveira, M.F. Marine debris ingestion and the use of diagnostic imaging in sea turtles: a review. Veterinární Medicína-Czech, 65(12): 511527, 2020.

Orós, J.; Torrent, A.; Calabuig, P.; Déniz, S. Diseases and causes of mortality among sea turtles stranded in the Canary Islands, Spain (1998-2001). Diseases of Aquatic Organisms, 63(1): 13-24, 2005.

Rizzi, M.; Rodrigues, F.L.; Medeiros, L.; Ortega, I.; Rodrigues, L.; Monteiro, D.S.; Kessler, F.; Proietti, M.C. Ingestion of plastic marine litter by sea turttle in southern Brazil: abundance, characteristics and potencial selectivity. Marine Pollution Bulletin, 140(1): 536-548, 2019.

Silva-Júnior, E.S.; Farias, D.S.D.; Bomfim, A.C.; Freire, A.C.B.; Revorêdo, R.A.; Rossi, S.;
Matushima, E.R.; Grisi-Filho, J.H.H.; Silva, F.J.L.; Gavilan, S.A. Stranded Marine Turtles in Northeastern Brazil: Incidence and spatialtemporal distribution of fibropapillomatosis.

Chelonian Conservation and Biology, 18(2): 249-258, 2019.

Smith, D.A.; Barker, I.K. Healing of cutaneous wounds in the common garter snake (Thamnophis sirtalis). Canadian Journal of Veterinary Research, 52(1): 111-119, 1988.

Spadola, F., Barillaro, G.; Morici, M.; Nocera, A.; Knotek, Z. The practical use of computed tomography in evaluation of shell lesions in six loggerhead turtles (Caretta caretta). Veterinární Medicína-Czech, 61(7): 394-398, 2016.

Stacy, N.I.; Bjorndal, K.A.; Perrault, J.R.; Martins, H.R.; Bolten, A.B. Blood analytes of oceanicjuvenile loggerhead sea turtles (Caretta caretta) from Azorean waters: reference intervals, sizerelevant correlations and comparisons to neritic loggerheads from western Atlantic coastal waters. Conservation Physiology, 6(1): 1-13, 2018.

Valente, A.L.S., Cuenca, R.; Zamora, M.; Parga, M.L.; Lavin, S.; Alegre, F.; Marco, I. Computed tomography of the vertebral column and coelomic structures in the normal loggerhead sea turtle (Caretta caretta). The Veterinary Journal, 174(2): 362-370, 2007. 